\title{
Fluid reasoning and the developing brain
}

\author{
Emilio Ferrer ${ }^{1}$, Elizabeth D. O'Hare ${ }^{2}$ and Silvia A. Bunge ${ }^{2,3 *}$ \\ 1 Department of Psychology, University of California, Davis, CA, USA \\ 2 Helen Wills Neuroscience Institute, University of California, Berkeley, CA, USA \\ ${ }^{3}$ Department of Psychology, University of California, Berkeley, CA, USA
}

Fluid reasoning is the cornerstone of human cognition, both during development and in adulthood. Despite this, the neural mechanisms underlying the development of fluid reasoning are largely unknown. In this review, we provide an overview of this important cognitive ability, the method of measurement, its changes over the childhood and adolescence of an individual, and its underlying neurobiological underpinnings. We review important findings from psychometric, cognitive, and neuroscientific literatures, and outline important future directions for this interdisciplinary research.

Keywords: problem-solving, intelligence, prefrontal cortex, rostrolateral parietal cortex, individual differences

\section{Edited by:}

Robert T. Knight, University

of California, Berkeley, CA, USA

Reviewed by:

Anna C. Nobre, University of Oxford, UK

Donald T. Stuss, University of Toronto,

Canada; Baycrest Centre for Geriatric

Care, Canada

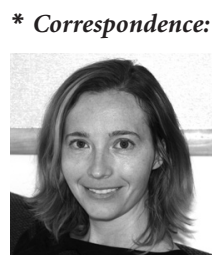

Silvia A. Bunge collaborated with Professor Emilio Ferrer at UC Davis to launch a longitudinal study on the normative development of fluid reasoning between the ages 6 and 18. Post-doctoral Fellow Elizabeth O'Hare and others have so far collected behavioral and brain imaging data pertaining to over 90 participants. Initial cross-sectional fMRI analyses for this project were reported in Wright et al., FiHN, 2007, and in Crone et al., Dev. Sci., 2009.

Structural analyses relating reasoning ability to cortical thickness and white matter coherence were presented at the Society for Neuroscience Annual Meeting in 2008.

sbunge@berkeley.edu

\section{INTRODUCTION}

Fluid reasoning (FR) is the capacity to think logically and solve problems in novel situations, independent of acquired knowledge (Cattell, 1987). It is an essential component of cognitive development (Goswami, 1992), as this capacity serves as a scaffold for children, in helping them acquire other abilities (Blair, 2006; Cattell, 1971, 1987). FR, in childhood, accurately predicts performance in school, university, and cognitively demanding occupations (Gottfredson, 1997). Despite this knowledge, we do not yet fully understand the cause for individual differences in fluid intelligence. This review examines the construct of FR, its development over childhood and adolescence, and its known underlying neurobiological mechanisms. We conclude by outlining the important challenges associated with this line of inquiry, and offering recommendations for future research.

\section{MEASUREMENT OF FR}

The term "fluid reasoning" was originally described in the Cattell's theory of fluid and crystallized intelligences. According to Cattell, FR - or fluid intelligence - referred to a general cognitive ability that emerges early in life and is applied by the child during any information retrieval process. Furthermore, FR greatly influences the way, in which children learn tasks that require complex spatial, numerical, or conceptual relations. Children add perceptual, discriminatory, and executive skills to their cognitive repertoire through experience. The complex abilities acquired are attached to particular perceptual and motor areas of the brain and become hardened, or "crystallized", abilities.

There are various measures adopted to assess FR. Of these measures, perhaps the most commonly used is the Raven's Progressive Matrices (RPM) test The RPM test requires participants to identify relevant features based on the spatial organization of an array of objects, and then select the object that matches one or more of the identified features (Figure 1A). The test measures relational reasoning, or the ability to consider one or more relationships between mental representations. As the number of relations increases in the RPM, participants tend to respond more slowly and less accurately. Like matrix reasoning tests, propositional analogy tests (Figure 1B) also evaluate relational reasoning, as it is necessary to determine whether the semantic relationship existing between two entities is the same as the relationship between two other, often completely different, entities. 
Fluid reasoning (FR)

The capacity to think logically

and solve problems in novel situations independent of acquired knowledge.

This construct is central to theories

of human intelligence.

Relational reasoning

A form of fluid reasoning consisting

of identifying correspondences

between the structures of distinct

mental representations.

Propositional analogy

A form of relational reasoning that entails the abstraction of a relationship between a familiar representation

and mapping it to a novel

representation (see Figure 1B).

Solving such a problem requires (1)

the abstraction of the relationship

between the base items (a bike moves

on the road), and (2) mapping

the relationship to the target pair

(a canoe moves on water).

Rostrolateral prefrontal cortex

\section{(RLPFC)}

Brain region corresponding to the lateral Brodmann area 10, which has been implicated in fluid reasoning, and in particular, relational integration.

Relational integration (or secondorder relational processing)

The cognitive process, by which several relations between mental representations are combined or compared, as in a 2-relational RPM problem, a propositional analogy, or a transitive inference problem. This is a critical component of relational reasoning that shows delayed maturation.

\section{FR DEVELOPMENT AND INDIVIDUAL DIFFERENCES DEVELOPMENTAL TRAJECTORY}

FR is believed to emerge in the first 2 or 3 years of life, after the development of general, perceptual, attentional and motoric capabilities (Cattell, 1987). Notably, FR follows a different developmental trajectory than crystallized abilities (McArdle et al., 2002), supporting the idea of separable cognitive functions (Horn, 1991; Schaie, 1996). The psychometric literature indicates that FR advances rapidly in early and middle childhood, continues to increase, though at a slower rate, until early adolescence, and reaches asymptotic values in the mid-adolescence to late-adolescence stage, after which it begins to decline (McArdle et al., 2002). Although agerelated changes in FR ability have been wellcharacterized, the mechanisms of such changes, especially the structure and function of brain areas underlying FR, are unknown.

\section{FR AND RELATED COGNITIVE ABILITIES}

FR has been linked to other important cognitive abilities. For example, cross-sectional behavioral studies indicate that FR is related to working memory and executive functioning (Engle et al., 1999), and to secondary memory (Mogle et al., 2008). Such studies provide information about the time-independent covariation between FR and other cognitive abilities. However, they do not reveal information about within-person changes and, more importantly, an empirical understanding of possible mechanisms underlying such covariation.

In longitudinal studies with adults, FR has been related to changes in crystallized abilities, shortterm memory, and processing speed (McArdle et al., 2000). Furthermore, among children and adolescents, FR has been identified to be a leading indicator of changes over time in crystallized abilities (McArdle, 2001) and changes in quantitative ability, academic knowledge, and reading (Ferrer and McArdle, 2004; Ferrer et al., 2007). In addition to these bottom-up and top-down influences, there are possible synergistic influences that involve working memory (Demetriou, 2002). This longitudinal research suggests that $F R$ is most closely related to processing speed and working memory, although other studies focused on the simultaneous changes of these constructs over time indicate a complex pattern of interrelations among variables, eliminating a simple interpretation of a single leading indicator of changes (McArdle et al., 2000).

\section{NEURAL BASIS OF FR}

An important endeavor for understanding FR is to identify the neural substrates that underlie such cognitive ability and its development (e.g., Duncan et al., 2000). Achieving the goal requires the usage of measures that directly map onto the theoretical construct of FR, such as the RPM task (Figure 1A).

Studies have demonstrated the importance of the frontal lobe in fluid intelligence (e.g., Duncan, 2005; Duncan et al., 1995). More specifically, functional Magnetic Resonance Imaging (fMRI) studies involving the RPM task in adults have demonstrated that a region in the anterior prefrontal cortex, known as the rostrolateral prefrontal cortex (RLPFC), is activated when participants engage in relational integration during RPM tasks (Christoff et al., 2001; Kroger et al., 2002). As RLPFC is activated more for 2-relational problems than 1- or 0 -relational problems, it appears that RLPFC is specifically engaged when participants must integrate across multiple mental representations (Ramnani and Owen, 2004). fMRI studies involving other visuospatial reasoning tasks have also linked RLPFC to the process of relational integration (Christoff et al., 2003; Smith et al., 2007). Additionally, in verbal propositional analogy tasks, RLPFC is preferentially engaged when participants must consider an analogy (identical in structure to the propositional analogy shown in Figure 1B, with words rather than pictures), as opposed to when participants must evaluate two individual semantic relationships (Bunge et al., 2005; Green et al., 2006; Wendelken et al., 2008).

In addition to RLPFC, the parietal cortex has been implicated in relational reasoning. Parietal activation has been shown to mediate the relationship between FR and performance during a demanding working memory task (Gray et al., 2003). Individuals with superior IQ scores rely more heavily on parietal cortex during relational integration tasks, compared to individuals with average IQ scores (Lee et al., 2006). Thus, it appears that while RLPFC is critical for relational integration during relational reasoning, the parietal cortex is essential for the identification and representation of visual-spatial relations that are fundamental to overall relational reasoning. The notion of parietal cortex as the "workhorse" of relational reasoning is consistent with a recent lab study, in which adults showed a higher degree of inferior parietal activation compared to children, during an RPM task (Crone et al., 2009). 


\section{A}

A

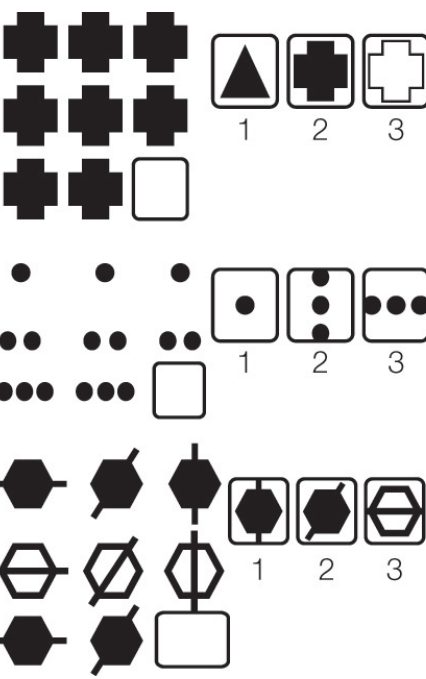

B
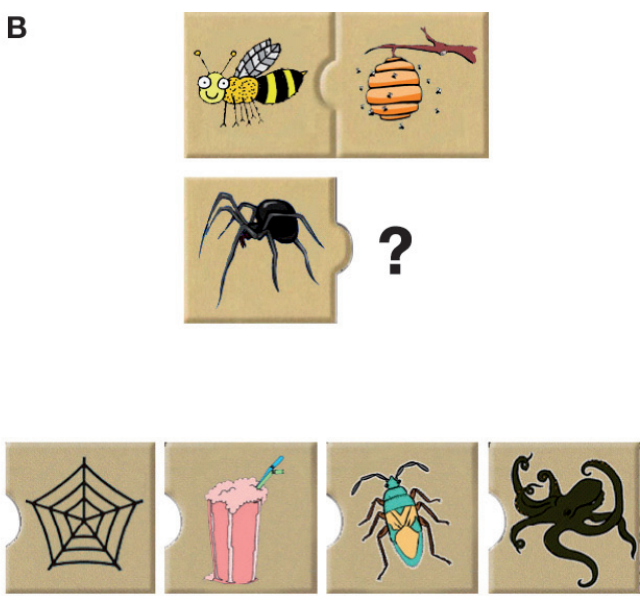

Figure 1 | (A) Sample matrix reasoning problem adapted from the RPM. Participants simply need to complete the array with the matching figure for 0-relational problems. Participants must identify a vertical or horizontal relationship between items in the array for 1-relational problems. For 2-relational problems, participants must jointly consider horizontal and vertical relations and hence, this task is considered to require relational integration abilities. The correct answers to the featured problems are choices 2, 3, and 1, respectively. (B) Sample propositional analogy task adapted from the Kaufman Brief Intelligence Test (KBIT). The correct answer is (A).

\section{CHANGES IN THE NEURAL SUBSTRATES OF FR OVER CHILDHOOD DEVELOPMENTAL CHANGES IN BRAIN STRUCTURE}

Structural brain development during late childhood and adolescence consists of concomitant reductions in synaptic density and increases in axonal myelination that proceeds along specific spatio-temporal patterns. Longitudinal MRI research confirms and extends prior postmortem work by demonstrating that in general, brain loss occurs first in the dorsal parietal lobes during childhood and then spreads anteriorly to dorsal frontal regions during adolescent and post-adolescent years (Gogtay et al., 2004; Shaw et al., 2008; Sowell et al., 2004). During this stage, RLPFC exhibits cortical thinning until the age of 20 years (O'Donnell et al., 2005).

In adults, IQ has been observed to correlate positively with cortical thickness in bilateral RLPFC (Narr et al., 2007). However, a longitudinal MRI study found that it was the trajectory of cortical thickness in aPFC in individuals from ages 4-29 rather than the actual values that distinguished highly intelligent individuals from others (Shaw et al., 2006). By this measure, the most intelligent children displayed a protracted increase in cortical thickness, followed by adolescents, in whom cortical thickness was observed to have undergone significant thinning (Shaw et al., 2006). If the authors had compared these individuals only at ages 8,10 , or 12 , they would have concluded that individuals of superior IQ exhibited lower, greater, or equivalent cortical thickness in aPFC, respectively, when compared with average or high-IQ individuals. In fact, their longitudinal data reveal that any one of these accounts would have been an incomplete and potentially misleading characterization of the differences between these groups of children. Similarly, we expect that the large, ongoing longitudinal study will shed additional light on the relationship between age, individual differences in FR ability, and cortical thickness.

\section{AGE-RELATED CHANGES IN BRAIN ACTIVATION ASSOCIATED WITH FR}

Three studies have examined the neural basis of FR in a pediatric sample (Crone et al., 2009; Eslinger et al., 2008; Wright et al., 2007). In the first of fMRI studies, the group (Wright et al., 2007) tested children (ages 6-13) and adults (ages 19-26) on a visual analogy task with semantic (1-relational problems) and analogy (2-relational problems) conditions. In semantic trials, participants were presented with one target image (e.g., a baseball) and five response images. They had to select the response image that best matched the target image (e.g., a baseball bat). In analogy problems, participants were presented with three target images and had to select one of the four response figures that completed the array (Figure 1B).

Among children, it was observed that RLPFC activation increases from the age of 6-13, bilater- 

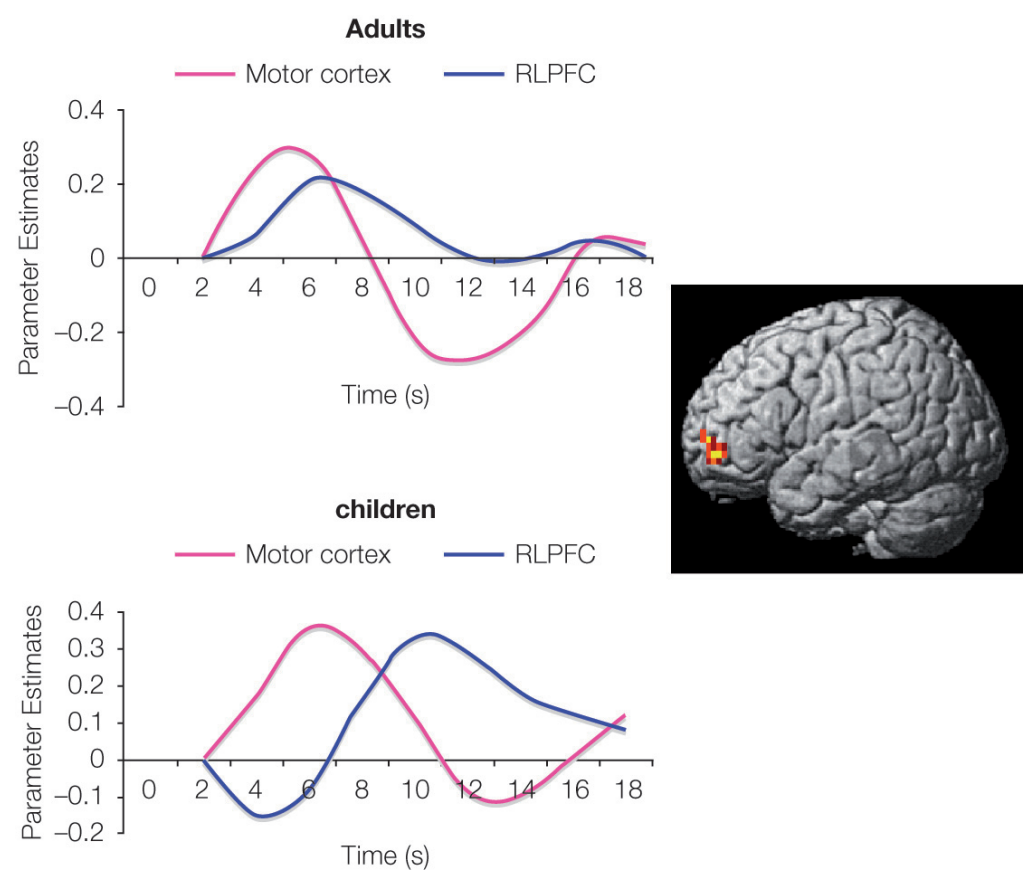

Figure 2 | During the performance of visual analogy problems, RLPFC activation (surface rendering shown on left) in children peaked after motor cortex. In other words, RLPFC was not engaged in time to influence the behavioral response on 2-relational problems.

ally for 1-relational problems and in left RLPFC for 2-relational problems (i.e., analogy problems). Among adults, it was found that individuals performing analogy problems with the greatest accuracy showed the largest differential recruitment of RLPFC during relational integration problems as compared with 1-relational problems. The findings suggest that RLPFC involvement in analogical reasoning may go through several developmental stages. During middle childhood, RLPFC is recruited during the performance of visual analogy tasks, but is not distinguished between 1-relational and 2-relational problems. In early adulthood, RLPFC shows selective engagement for the processing and integration of multiple relations (e.g., relational integration). Furthermore, time series analyses have revealed delayed RLPFC activation in children, compared to adults (Figure 2).

Arguing against the possibility that children merely display sluggish hemodynamic response in RLPFC, similar time courses have been observed in the region in the age group of 8-12 years - as well as in young adults, in the context of a RPM task (Crone et al., 2009). Furthermore, a study comparing the hemodynamic response between children and adults has not revealed consistent differences in timing between these groups (Kang et al., 2003). Hence, the shift in the timing of RLPFC activation is task-specific: children engage RLPFC in a timely manner on the RPM task, but not on the visual analogy task. It is speculated that children rely on their knowledge about the objects depicted in the visual analogy problems, rather than approaching the problems analytically. A line of research shows that sometimes, even adults endorse illogical lines of reasoning if the content, about which the adults are asked to reason, is familiar and plausible (Braine, 1978). Similarly, it may be true that children approach visual analogy problems in the inappropriate way because they can access semantic memory for objects comprising the problem. However, this is not probable for RPM problems, which are largely devoid of semantic information.

These observations are consistent with the possibility that children tend to respond too hastily, and that the performance of children can benefit from training on the tasks requiring response inhibition. Additionally or alternatively, training on FR tasks might lead to more efficient relational integration.

The idea of a developmental shift in the networks of the brain regions involved in relational integration, across childhood and adolescence, receives additional support from the study involving an RPM-type task (Crone et al., 2009). In adults, RLPFC did not discriminate between 0 -relational and 1-relational problems and was 


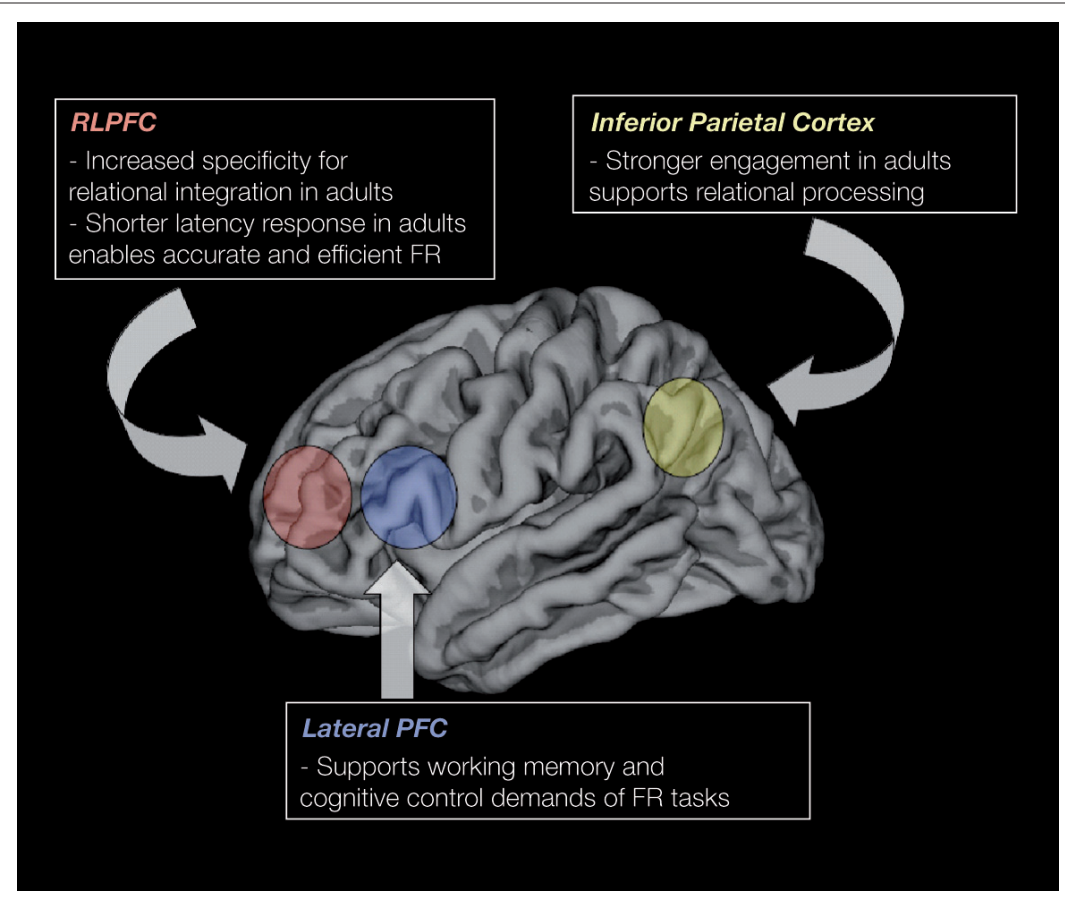

Figure 3 | Schematic illustration of some of the key regions that support FR, and data in relation to their change in the regions over middle childhood and adolescence phases.

specifically recruited with dorsolateral prefrontal cortex (DLPFC) and posterior parietal cortex for 2-relational problems. In contrast, children aged 8-12 recruited both RLPFC and DLPFC to similar extents for 1- and 2-relational problems. These findings are also consistent with a recent report that indicates decreased activation with age, in bilateral RLPFC and DLPFC, during 1-relational problem solving (Eslinger et al., 2008).

Altogether, the results indicate that while regions that support relational reasoning are already engaged by middle childhood, the precise ways in which the regions contribute to reasoning are fine-tuned via structural brain changes during adolescence (see Figure 3).

\section{CURRENT AND FUTURE DIRECTIONS}

Neuroscientific research has begun to provide clues about the changes in brain function underlying developmental changes in FR. However, there are still many unknown changes. First, it is necessary to measure the reasoning-related patterns of brain activation when reasoning ability first begins to emerge in young childhood and changes most rapidly (e.g., Goswami, 1992). Marked changes have been observed in performance between the age groups 4-6 and 7-10 on the visual analogy task (B. Matlen, unpublished undergraduate honors thesis). By the age of 6 years, the youngest age at which fMRI data were successfully collected on the reasoning tasks, children had already begun to reason - although this ability improved further over subsequent development.

Second, longitudinal fMRI data are needed to examine within-subject changes in brain function, which underlie improvements in FR. Indeed, the longitudinal structural MRI data from Shaw et al. (2006) revealed that it was the trajectory of cortical thickening and its thinning over time that distinguished individuals on the basis of IQ, rather than the thickness values themselves. Similarly, the ongoing longitudinal fMRI study is expected to reveal new insights into the developmental changes in brain function underlying FR.

Third, a longitudinal approach enables modeling of the complex patterns of interrelations between the cognitive abilities that contribute to $\mathrm{FR}$, including processing speed, working memory, and specific executive functions. These behavioral measures, as well as brain measures, can be evaluated with regard to their predictive value the extent to which a combination of these measures at one timepoint allows the prediction of an individual's FR ability at a later time (Hoeft et al., 2007)

Fourth, it is important to examine the implications of the research for school achievement. It is necessary to determine whether a deeper understanding of the mechanisms underlying FR development will enable the development of an effective intervention for children who struggle to perform well in school as a result of low FR ability. Encouraging preliminary evidence from the laboratory indicates that 8 weeks of training on FR - but not on processing speed - leads to improved performance on standard reasoning tasks in children aged 7-9 years, some of whom had low IQ scores at the outset of training. It will be necessary to replicate these findings in a larger sample, and test whether FR training has a positive and lasting influence on school performance.

\section{ACKNOWLEDGMENTS}

The authors thank Samantha Wright, Bryan Matlen, and Carol Baym for their contributions to the empirical paper in Frontiers in Human Neuroscience, on which this focused review was based. They also thank Brian Johnson, Kirstie Whitaker, Zdena Op de Macks, Allyson Mackey, Susanna Hill, Ori Elis, Eveline Crone, Carter Wendelken, and other current and former members of the Bunge laboratory for their work on the longitudinal study of reasoning development, funded by NIMH grant R01NS57146-01. 


\section{REFERENCES}

Blair, C. (2006). How similar are fluid cognition and general intelligence? A developmental neuroscience perspective on fluid cognition as an aspect of human cognitive ability. Behav. Brain Sci. 26, 109-160.

Braine, M. D. S. (1978). On the relations between the natural logic of reasoning and standard logic. Psychol. Rev. 85, 1-21.

Bunge, S. A., Wendelken, C., Badre, D., and Wagner, A. D. (2005). Analogical reasoning and prefrontal cortex: evidence for separable retrieval and integration mechanisms. Cereb. Cortex 15, 239-249.

Cattell, R. B. (1971). Abilities: Their Structure, Growth and Action. Boston, Houghton-Mifflin.

Cattell, R. B. (1987). Intelligence: Its Structure, Growth and Action. Amsterdam, North-Holland.

Christoff, K., Prabhakaran, V., Dorfman, J., Zhao, Z., Kroger, J. K., Holyoak, K. J., and Gabrieli, J. D. (2001). Rostrolateral prefrontal cortex involvement in relational integration during reasoning. Neuroimage 14, 1136-1149.

Christoff, K., Ream, J. M., Geddes, L. P., and Gabrieli, J. D. (2003). Evaluating self-generated information: anterior prefrontal contributions to human cognition. Behav. Neurosci. 117, 1161-1168.

Crone, E. A., Wendelken, C., van Leijenhorst, L., Honomichl, R. D., Christoff, K., and Bunge, S. A. (2009). Neurocognitive development of relational reasoning. Dev. Sci. 12, 55-66.

Demetriou, A. (2002). The Development of Mental Processing: Efficiency, Working Memory, and Thinking. Monographs of the Society for Research in Child Developent 67, Serial No. 268.

Duncan, J. (2005). Frontal lobe function and general intelligence: why it matters. Cortex 41, 215-217.

Duncan, J., Burgess, P., and Emslie, H. (1995). Fluid intelligence after frontal lobe lesions. Neuropsychologia 33, 261-268.

Duncan, J., Seitz, R. J., Kolodny, J., Bor, D., Herzog, H., Ahmed, A., Newell, F. N., and Emslie, H. (2000). A neural basis for general intelligence. Science 289, 457-460.

Engle, R.W., Kane,M.J., and Tuholski, S. W. (1999). Individual differences in working memory capacity and what they tell us about controlled attention, general fluid intelligence, and functions of the prefrontal cortex. In Models of Working Memory, A. Miyake and P. Shah, eds (New York, Cambridge University Press), pp. 102-134.
Eslinger,P.J.,Blair,C.,Wang, J.,Lipovsky, B., Realmuto, J., Baker, D., Thorne, S., Gamson, D., Zimmerman, E., Rohrer, L., and Yang, Q. X. (2008). Developmental shifts in $\mathrm{PMRI}$ activations during visuospatial relational reasoning. Brain Cogn. 69, 1-10.

Ferrer, E., and McArdle, J. J. (2004). An experimental analysis of dynamic hypotheses about cognitive abilities and achievement from childhood to early adulthood. Dev. Psychol. 40, 935-952.

Ferrer, E., McArdle, J. J., Shaywitz, B. A., Holahan, J. N., Marchione, K., and Shaywitz, S. E. (2007). Longitudinal models of developmental dynamics between reading and cognition from childhood to adolescence. Dev. Psychol. 43, 1460-1473.

Gogtay, N., Giedd, J. N., Lusk, L., Hayashi, K. M., Greenstein, D., Vaituzis, A. C., Nugent, T. F., 3rd, Herman, D. H., Clasen, L. S., Toga, A. W., Rapoport, J. L., and Thompson, P. M. (2004). Dynamic mapping of human cortical development during childhood through early adulthood. Proc. Natl. Acad. Sci. U.S.A. 101, 8174-8179.

Goswami, U.(1992).Analogical Reasoning in Children. Hillsdale, NJ, Lawrence Erlbaum.

Gottfredson, L. S. (1997). Why g matters: the complexity of everyday life. Intelligence 24, 79-132.

Gray, J. R., Chabris, C. F., and Braver, T. S. (2003). Neural mechanisms of general fluid intelligence. Nat. Neurosci. 6, 316-322.

Green,A.E., Fugelsang, J.A., Kraemer, D. J., Shamosh, N. A., and Dunbar, K. N. (2006). Frontopolar cortex mediates abstract integration in analogy. Brain Res. 1096, 125-137.

Hoeft, F., Ueno, T., Reiss, A. L., Meyler, A., Whitfield-Gabrieli, S., Glover, G. H., Keller, T.A., Kobayashi, N., Mazaika, P., Jo, B., Just, M. A., and Gabrieli, J. D. (2007). Prediction of children's reading skills using behavioral, functional, and structural neuroimaging measures. Behav. Neurosci. 121, 602-613.

Horn, J. L. (1991). Measurement of intellectual capabilities: a review of theory. In: Woodcock-Johnson Technical Manual, K. S. McGrew, J.K. Werder, and R. W. Woodcock, eds (Allen, TX, DLM Teaching Resources), pp. 197-246.

Kang, H. C., Burgund, E. D., Lugar, H. M., Petersen, S. E., and Schlaggar, B. L. (2003). Comparison of functional activation foci in children and adults using a common stereotactic space. Neuroimage 19, 16-28.

Kroger, J. K., Sabb, F. W., Fales, C. L., Bookheimer, S. Y., Cohen, M. S., and Holyoak, K. J. (2002). Recruitment of anterior dorsolateral prefrontal cor- tex in human reasoning: a parametric study of relational complexity. Cereb. Cortex 12, 477-485.

Lee, K. H., Choi, Y.Y., Gray, J. R., Cho, S. H., Chae, J.H., Lee, S., and Kim, K. (2006) Neural correlates of superior intelligence: stronger recruitment of posterior parietal cortex. Neuroimage 29, 578-586.

McArdle, J. J. (2001). A latent difference score approach to longitudinal dynamic structural analysis. In Structural Equation Modeling: Present and Future. A Festschrift in Honor of Karl Joreskog, R. Cudeck, S. du Toit, and D. Sorbom, eds (Lincolnwood IL, Scientific Software International), pp. 341-380.

McArdle, J. J., Ferrer-Caja, E., Hamagami, F., and Woodcock, R. W. (2002). Comparative longitudinal structural analysis of growth and decline of multiple intellectual abilities over the lifespan. Dev. Psychol. 38, 113-142.

McArdle, J. J., Hamagami, F., Meredith, W., and Bradway, K. P. (2000). Modeling the dynamic hypotheses of Gf-Gc theory using longitudinal life-span data. Learn. Individ. Differ. 12, 53-79.

Mogle, J. A., Lovett, B. J., Stawski, R. S. and Sliwinski, M. J. (2008). What's so special about working memory? An examination of the relationships among working memory, secondary memory, and fluid intelligence. Psychol. Sci. 19, 1071-1077.

Narr, K. L., Woods, R. P., Thompson, P. M. Szeszko,P., Robinson,D.,Dimtcheva, T., Gurbani, M., Toga, A. W., and Bilder, R. M. (2007). Relationships between IQ and regional cortical gray matter thickness in healthy adults. Cereb. Cortex 17, 2163-2171.

O'Donnell, S., Noseworthy, M. D., Levine, B., and Dennis, M. (2005). Cortical thickness of the frontopolar area in typically developing children and adolescents. Neuroimage 24 948-954.

Ramnani, N., and Owen, A. M. (2004) Anterior prefrontal cortex: insights into function from anatomy and neuroimaging. Nat. Rev. Neurosci. 5 , 184-194.

Schaie, K. W. (1996). Intellectual Development in Adulthood: The Seattle Longitudinal Study. New York, Cambridge University Press.

Shaw, P., Greenstein, D., Lerch, J., Clasen, L., Lenroot, R., Gogtay, N., Evans, A., Rapoport, J., and Giedd, J. (2006). Intellectual ability and cortical development in children and adolescents. Nature 440, 676-679.

Shaw, P., Kabani, N. J., Lerch, J. P., Eckstrand, K., Lenroot, R., Gogtay, N., Greenstein, D., Clasen, L., Evans, A., Rapoport, J. L., Giedd, J. N., and
Wise, S. P.(2008).Neurodevelopmental trajectories of the human cerebral cortex. J. Neurosci. 28, 3586-3594.

Smith, R., Keramatian, K., and Christoff, K. (2007). Localizing the rostrolateral prefrontal cortex at the individual level. Neuroimage 36, 1387-1396.

Sowell, E. R., Thompson, P. M., Leonard, C. M., Welcome, S. E., Kan, E. and Toga, A. W. (2004). Longitudinal mapping of cortical thickness and brain growth in normal children. J. Neurosci. 24, 8223-8231.

Wendelken, C., Nakhabenko, D., Donohue, S. E., Carter, C. S., and Bunge, S. A. (2008). "Brain is to thought as stomach is to?": investigating the role of rostrolateral prefrontal cortex in relational reasoning. J. Cogn. Neurosci. 20, 682-693.

Wright, S. B., Matlen, B. J., Baym, C. L., Ferrer, E., and Bunge, S. A. (2007). Neural correlates of fluid reasoning in children and adults. Front. Hum. Neurosci. 1, 8 .

Conflict of Interest Statement: The authors declare that the research was conducted in the absence of any commercial or financial relationships that could be construed as a potential conflict of interest.

Received: 15 January 2009; paper pending published: 09 February 2009; accepted: 26 February 2009; published: 01 May 2009.

Citation: Front. Neurosci. (2009) 3,1: 46-51. doi: 10.3389/neuro.01.003.2009

Copyright (c) 2009 Ferrer, O'Hare and Bunge. This is an open-access article subject to an exclusive license agreement between the authors and the Frontiers Research Foundation, which permits unrestricted use, distribution, and reproduction in any medium, provided the original authors and source are credited. 